\title{
Surveillance of COVID-19- Associated Multisystem Inflammatory Syndrome in Children, South Korea
}

\author{
Young June Choe, Eun Hwa Choi, Jong Woon Choi, Byung Wook Eun, Lucy Youngmin Eun, \\ Yae-Jean Kim, Yeo Hyang Kim, Young A. Kim, Yun-Kyung Kim, Ji Hee Kwak, Hyuk Min Lee, Hyunju Lee, \\ Joon Kee Lee, June Dong Park, Eun-Jin Kim, Young Joon Park, Jin Gwack, Sang Won Lee
}

A concerning development during the coronavirus disease pandemic has been multisystem inflammatory syndrome in children. Reports of this condition in East Asia have been limited. In South Korea, 3 cases were reported to the national surveillance system for multisystem inflammatory syndrome in children. All casepatients were hospitalized and survived with no major disease sequelae.

mid the coronavirus disease (COVID-19) panAdemic, multisystem inflammatory syndrome in children (MIS-C) has emerged as a major concern globally (1). MIS-C features clinical characteristics that overlap with Kawasaki disease, including high fever, mucocutaneous involvement, and affecting of coronary arteries. Yet, reports of MIS-C have been limited in East Asia countries, where the incidence of Kawasaki disease is high (2).

Although South Korea was one of the countries struck early in the COVID-19 pandemic, spread of the virus there has been relatively contained. However,

Author affiliations: Korea University Anam Hospital, Seoul, South Korea (Y.J. Choe); Seoul National University College of Medicine, Seoul (E.H. Choi, H. Lee, J.D. Park); Bundang Jesaeng General Hospital, Seongnam, South Korea (J.W. Choi); Eulji University School of Medicine, Seoul (B.W. Eun); Yonsei University College of Medicine, Seoul (L.Y. Eun, H.M. Lee); Sungkyunkwan University School of Medicine, Seoul (Y.-J. Kim, J.H. Kwak); School of Medicine Kyungpook National University, Daegu, South Korea (Y.H. Kim); Pusan National University Children's Hospital, Yangsan, South Korea (Y.A. Kim); Korea University College of Medicine, Seoul (Y.-K. Kim); Chungbuk National University Hospital, Cheongju, South Korea (J.K. Lee); Korea Disease Control and Prevention Agency, Cheongju (E.-J. Kim, Y.J. Park, J. Gwack, S.W. Lee)

DOI: https://doi.org/10.3201/eid2704.210026 reports on MIS-C from other countries has necessitated the monitoring of COVID-19-associated MIS-C at the national level. In May 2020, the Korean Society of Pediatric Infectious Diseases, Korean Society of Kawasaki Disease, and Korean Society of Pediatric Critical Care Medicine, with support from the Korea Disease Control and Prevention Agency, created a strategic framework for prospective surveillance of MIS-C in South Korea. In this study, we describe the development of the MIS-C surveillance system and report the clinical characteristics of children meeting the case definition of MIS-C in South Korea.

\section{The Study}

First, the Case Assessment Committee (CAC) was established, consisting of 4 pediatric infectious disease specialists, 3 pediatric cardiologists, 3 pediatric intensivists, 1 clinical microbiologist, and 1 epidemiologist. A case reporting form was created, and members of the Korean Pediatric Society $(n=5,891)$ were contacted to provide assistance with data collection and reporting.

Once a suspected MIS-C case was reported, CAC members quickly assessed whether the case met the clinical criteria for MIS-C case definition. In accordance with the Infectious Disease Control and Prevention Act (chapter 4, article 18), the public health officers then conducted an epidemiologic investigation of all suspected MIS-C cases. For all reported cases, the Korea Disease Control and Prevention Agency performed serologic assays for severe acute respiratory syndrome coronavirus 2 (SARS-CoV-2), including neutralizing antibody tests and the Anti-SARS-CoV-2 ELISA Assay for detection of IgG (EUROIMMUN, https://www.euroimmun.com). CAC meetings were held on an ad hoc basis for case ascertainment, 
treatment consultation, and exchange of knowledge. The study was approved by the Institutional Review Board of Seoul National University Hospital (approval no. 2012-136-118).

During May-November 2020, a total of 2,287 COVID-19 cases in persons 0-19 years of age were reported (Figure). During the surveillance period, 9 suspected cases of MIS-C were reported to the surveillance system. Of the reported cases, $3(33 \%)$ casepatients had evidence of COVID-19 exposure (positive for SARS-CoV-2 by PCR, SARS-CoV-2 antibody detection, or exposure history), and their illness was assessed as COVID-19-associated MIS-C, which likely occurred 3-4 weeks after the diagnosis of COVID-19 (Table).

The age of case-patients ranged from 11 to 14 years, 2 were boys, and none had preexisting conditions. All case-patients had fever and abdominal symptoms (abdominal pain, nausea, vomiting, or diarrhea) at admission. Mucocutaneous symptoms and signs (mucosal changes, skin rash, extremity changes) occurred in 2 patients, and all patients had documented hypotension $(<50$ th percentile, adjusted for age, sex, and height). All case-patients had marked leukocytosis or elevated inflammatory markers. Echocardiography showed coronary artery dilatation (z-scores 1.64-3.98 mm for left coronary arteries), mitral regurgitation, or left ventricular dysfunction. Chest radiography or computed tomography showed pulmonary edema or pleural effusion. Abdominal ultrasound or computed tomography showed mesenteric lymphadenopathies, hyperechoic liver, or hypertrophic gall bladder. All 3 case-patients received intravenous immunoglobulin (IVIg); 1 patient (case 3) received methylprednisolone pulse therapy and immunomodulatory agent (Anakinra) because of persistent hypotension after initial IVIg treatment. Two patients received inotropic agents and required transfer to the intensive care unit (ICU), but no patients required mechanical ventilation. The duration of hospitalization was 10-19 days, and duration of ICU admission was 6-7 days. All 3 patients received aspirin and have survived to date with no major disease sequelae.

\section{Conclusions}

We describe MIS-C surveillance results from South Korea, an East Asia country with high incidence of Kawasaki disease. As of December 15, 2020, COVID-19 had been diagnosed in 4,107 children and adolescents 0-19 years of age in South Korea, which translates roughly to $0.07 \%$ of all childhood COVID-19 cases reported in South Korea (3). Concern about MIS-C was raised after episodes of increased incidence of Kawasaki-like disease were noted in children after COVID-19 diagnosis in Europe and the United States $(4,5)$. In South Korea, there was no substantial increase in Kawasaki disease-related hospitalizations in 2020 compared with 2016-2019 (6). There might be ethnic differences in susceptibility; only $5 \%$ of MIS-C cases in New York (USA) occurred in Asian persons (7). Reports from India (8), Pakistan (9), and Iran (10) underscore the importance of monitoring MIS-C cases; however, surveillance data have not yet been reported for East Asia countries. Alongside genetic susceptibility, the

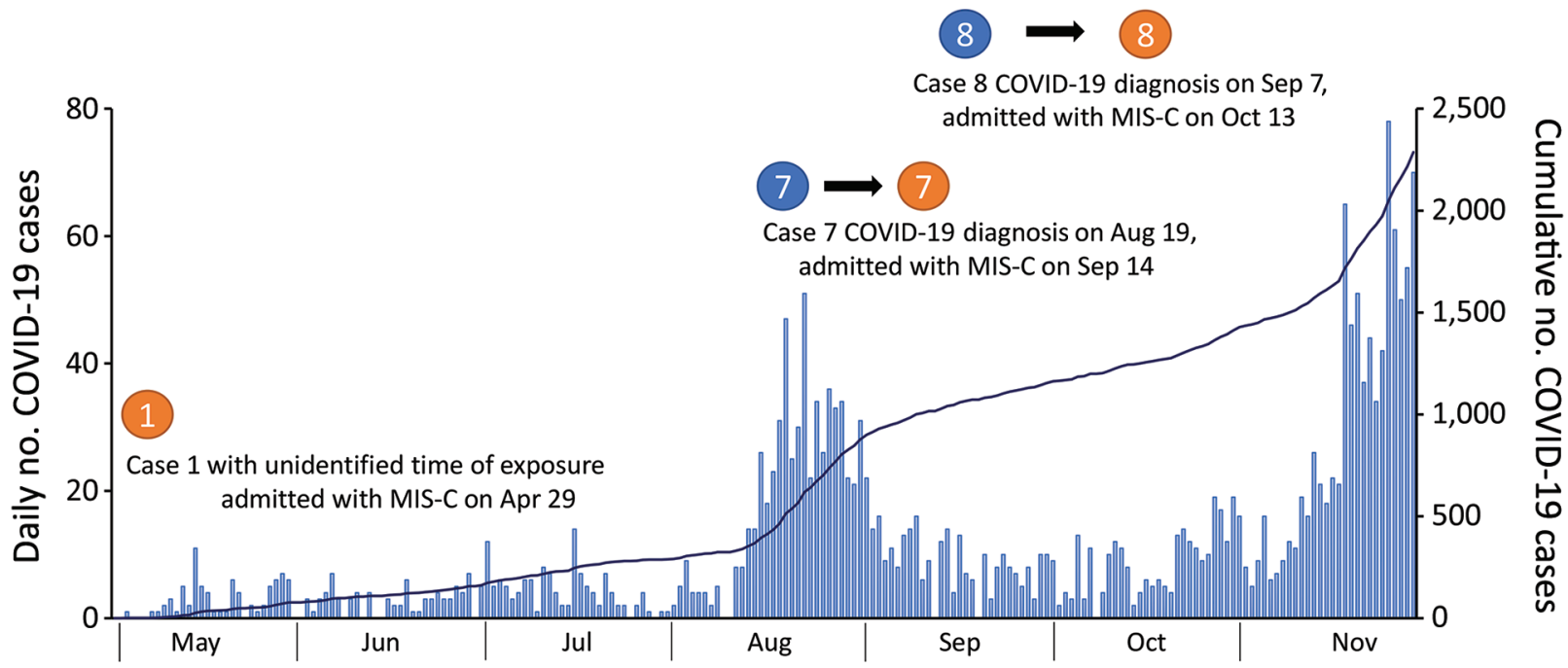

Figure. Daily number (bars) and cumulative number (line) of COVID-19 cases among children 0-19 years of age, South Korea, MayNovember 2020. The occurrences of the 3 cases of multisystem inflammatory syndrome are indicated. COVID-19, coronavirus disease; MIS-C, multisystem inflammatory syndrome in children. 
Table. Demographics, clinical features, treatments, and outcomes of the 3 COVID-19-associated MIS-C case-patients, South Korea, May-November 2020*

\begin{tabular}{|c|c|c|c|}
\hline Characteristics & Case 1 & Case 2 & Case 3 \\
\hline Age, y & 11 & 11 & 14 \\
\hline Sex & Boy & Boy & Girl \\
\hline Underlying disease & None & None & None \\
\hline \multicolumn{4}{|l|}{ Clinical signs and symptoms } \\
\hline Initial symptoms & Fever, abdominal pain & $\begin{array}{c}\text { Fever, abdominal pain, } \\
\text { headache, nausea, vomiting }\end{array}$ & $\begin{array}{l}\text { Fever, abdominal pain, } \\
\text { diarrhea }\end{array}$ \\
\hline Fever & Present & Present & Present \\
\hline Conjunctival injection & Present & Present & Present \\
\hline Mucosal change & Present & None & Present \\
\hline Skin rash & Present & None & Present \\
\hline Extremity changes & Present & None & Present \\
\hline Lymphadenopathy & None & None & None \\
\hline Gastrointestinal symptoms & Present & Present & Present \\
\hline Hypotension & Present & Present & Present \\
\hline \multicolumn{4}{|l|}{ Inflammatory markers (peak) } \\
\hline 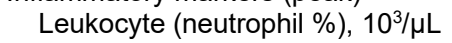 & $7.55(87)$ & $9.55(82.8)$ & $26.56(93)$ \\
\hline $\mathrm{ESR}, \mathrm{mm} / \mathrm{h}$ & NT & 82 & 77 \\
\hline $\mathrm{CRP}, \mathrm{mg} / \mathrm{L}$ & 18.95 & 10.36 & $>30$ \\
\hline Fibrinogen, mg/dL & 633 & NT & NT \\
\hline Procalcitonin, $\mathrm{ng} / \mathrm{mL}$ & 14.55 & 1.54 & 9.62 \\
\hline D-dimer, $\mu \mathrm{g} / \mathrm{mL}$ & 894 & 2.5 & 3.95 \\
\hline Ferritin, $\mu \mathrm{g} / \mathrm{mL}$ & NT & 2485 & 663 \\
\hline IL-6, pg/mL & NT & NT & 2410 \\
\hline
\end{tabular}

Abnormal imaging studies

Echocardiography

Chest radiography or CT

Abdominal ultrasound or CT
Coronary dilatation

Bilateral pleural effusion, pneumonic infiltration

Abdominal

lymphadenopathy

Mitral regurgitation
Suspected pulmonary
edema
Mesenteric
lymphadenopathy

Mitral regurgitation

Coronary dilatation, left ventricle dysfunction

Bilateral pulmonary edema, pleural effusion Hyperechoic liver, gallbladder hypertrophic edema, peripancreatic

fluids, splenomegaly, scant pelvic ascites

\begin{tabular}{|c|c|c|c|}
\hline \multicolumn{4}{|l|}{ Treatment } \\
\hline IVIg & Provided & Provided & Provided \\
\hline ASA & Provided & Provided & Provided \\
\hline Steroids & Not provided & Not provided & Provided \\
\hline Immunomodulatory & Not provided & Not provided & Provided (Anakinra) \\
\hline Inotropic agent & Provided & Not provided & Provided \\
\hline ICU care & Provided & Not provided & Provided \\
\hline Mechanical ventilator & Not provided & Not provided & Not provided \\
\hline \multicolumn{4}{|l|}{ Outcome } \\
\hline Hospitalization, d & $12 \mathrm{~d}$ & $10 d$ & $19 d$ \\
\hline ICU admission, $\mathrm{d}$ & $6 \mathrm{~d}$ & NA & $7 \mathrm{~d}$ \\
\hline Prognosis & Improved, discharged & Improved, discharged & Improved, discharged \\
\hline \multicolumn{4}{|c|}{$\begin{array}{l}\text { *MIS-C clinical case definition is as follows: age } \leq 19 \text { y, fever } \geq 38.0^{\circ} \mathrm{C} \text { for } \geq 24 \mathrm{~h} \text {, laboratory evidence of inflammation (i.e., elevation of ESR, CRP, } \\
\text { fibrinogen, procalcitonin, d-dimer, ferritin, LDH, IL-6, neutrophilia, lymphopenia, hypoalbuminemia), multisystem involvement ( } \geq 2 \text { organ systems involved), } \\
\text { severe illness requiring hospitalization, and no other plausible microbial cause of inflammation (i.e., bacterial sepsis, staphylococcal/streptococcal toxic } \\
\text { shock syndromes, enteroviral myocarditis). Evidence of SARS-CoV-2 exposure history defined as positive SARS-CoV-2 by RT-PCR, positive serology } \\
\text { (neutralizing antibody or anti-SARS-CoV-2 IgG), or exposure to individual with COVID-19 } \leq 4 \text { weeks before onset of symptoms (epidemiologic linkage with } \\
\text { individual or cluster). ASA, acetylsalicylic acid; COVID-19, coronavirus disease 2; CRP, C-reactive protein; CT, computed tomography; ESR, erythrocyte } \\
\text { sedimentation rate; ICU, intensive care unit; IL-6, interleukin 6; IVIg, intravenous immunoglobulin; LDH, lactate dehydrogenase; MIS-C, multisystem } \\
\text { inflammatory syndrome in children; NA, not applicable; NT, not tested; RT-PCR, reverse transcription PCR; SARS-CoV-2, severe acute respiratory } \\
\text { syndrome coronavirus 2. }\end{array}$} \\
\hline
\end{tabular}

background incidence of SARS-CoV-2 infection might play a critical role in the occurrence of MIS-C. Although estimates of risk for MIS-C after SARSCoV-2 infection are not yet available, we report a rough estimate in South Korea, where COVID-19 testing is widely accessible (11). Our findings suggest that the incidence of MIS-C is low among children with COVID-19 in this country. However, COVID-19-as- sociated MIS-C might cause serious clinical outcomes requiring ICU care and might require immunomodulatory agents.

All 3 MIS-C case-patients experienced gastrointestinal symptoms, which is consistent with reports from Italy (5), the United States (12), and the United Kingdom (13) that indicate gastrointestinal symptoms appear to be the most prominent 
clinical manifestation of MIS-C. Gastrointestinal involvement might also be a predictor of severe $\mathrm{CO}$ VID-19. A systematic review of 83 studies showed that diarrhea (odds ratio 1.50, 95\% CI 1.10-2.03; $\mathrm{p}=$ 0.01 ) was observed more often in patients with severe COVID-19 compared with patients with nonsevere COVID-19 (14). Previously, syndromic involvement of the gastrointestinal system has been associated with higher risk for IVIg resistance and coronary aneurysms in patients with Kawasaki disease (15). These features indicate the possibility of a mechanism linking gastrointestinal involvement and syndromic features for MIS-C and Kawasakilike illness, which needs further elucidation.

The first limitation of this study is that, given the intrinsic properties of a passive surveillance system, only a fraction of actual MIS-C cases might have been reported. Pediatricians are more likely to report cases that result in serious conditions; nonetheless, the case definition included hospitalization. Second, a large proportion of SARS-CoV-2 infections in children are asymptomatic; therefore, passive surveillance that relies on the presence of symptoms might underestimate the actual incidence of MIS-C. Despite these limitations, this study suggests that enhanced passive surveillance, including frequent outreach to pediatricians through academic societies, was a manageable scheme to monitor MIS-C in South Korea. Given that the level of SARS-CoV-2 community transmission was low during the surveillance period, passive surveillance was considered a robust plan to capture MIS-C cases at a national level.

Despite the introduction of vaccines, the global COVID-19 pandemic could continue for months. Therefore, surveillance is a critical tool for the detection and evaluation of serious complications in vulnerable population. Our experience offers a possible surveillance model for other countries concerned about COVID-19-associated MIS-C. MIS-C surveillance data in South Korea call for enhanced monitoring through syndromic and laboratory-based combination surveillance approaches.

\section{Acknowledgments}

We thank members of the Korean Pediatric Society who were involved in direct clinical service to children. We also thank public health officials for their cooperation and critical feedback.

\section{About the Author}

Dr. Choe is a pediatrician specializing in infectious diseases and epidemiology. His research addresses quantifying of and understanding the mechanisms of immunization programs' impact on public health, focusing on studies of respiratory virus transmission in the community and the effectiveness of control measures.

\section{References}

1. Kaushik A, Gupta S, Sood M, Sharma S, Verma S. A systematic review of multisystem inflammatory syndrome in children associated with SARS-CoV-2 infection. Pediatr Infect Dis J. 2020;39:e340-6. https://doi.org/10.1097/ INF.0000000000002888

2. Singh S, Vignesh P, Burgner D. The epidemiology of Kawasaki disease: a global update. Arch Dis Child. 2015;100:1084-8. https:// doi.org/10.1136/archdischild- 2014-307536

3. Korea Disease Control and Prevention Agency. Coronavirus disease-19 dashboard [cited 2020 Jan 5]. http:/ / ncov.mohw. go.kr/en/

4. Dufort EM, Koumans EH, Chow EJ, Rosenthal EM, Muse A, Rowlands J, et al.; New York State and Centers for Disease Control and Prevention Multisystem Inflammatory Syndrome in Children Investigation Team. Multisystem inflammatory syndrome in children in New York State. N Engl J Med. 2020;383:347-58. https:/ / doi.org/10.1056/ NEJMoa2021756

5. Verdoni L, Mazza A, Gervasoni A, Martelli L, Ruggeri M, Ciuffreda M, et al. An outbreak of severe Kawasaki-like disease at the Italian epicentre of the SARS-CoV-2 epidemic: an observational cohort study. Lancet. 2020;395:1771-8. https://doi.org/10.1016/S0140-6736(20)31103-X

6. Kim YJ, Park H, Choi YY, Kim YK, Yoon Y, Kim KR, et al. Defining association between COVID-19 and the multisystem inflammatory syndrome in children through the pandemic. J Korean Med Sci. 2020;35:e204. https://doi.org/10.3346/jkms.2020.35.e204

7. Lee EH, Kepler KL, Geevarughese A, Paneth-Pollak R, Dorsinville MS, Ngai S, et al. Race/ethnicity among children with COVID-19-associated multisystem inflammatory syndrome. JAMA Netw Open. 2020;3:e2030280. https://doi.org/10.1001/jamanetworkopen.2020.30280

8. Jain S, Sen S, Lakshmivenkateshiah S, Bobhate P, Venkatesh S, Udani S, et al. Multisystem inflammatory syndrome in children with COVID-19 in Mumbai, India. Indian Pediatr. 2020;57:1015-9. https://doi.org/10.1007/ s13312-020-2026-0

9. Clark BC, Sanchez-de-Toledo J, Bautista-Rodriguez C, Choueiter N, Lara D, Kang H, et al. Cardiac abnormalities seen in pediatric patients during the SARS-CoV2 pandemic: an international experience. J Am Heart Assoc. 2020;9:e018007. https://doi.org/10.1161/JAHA.120.018007

10. Mamishi S, Movahedi Z, Mohammadi M, Ziaee V, Khodabandeh M, Abdolsalehi MR, et al. Multisystem inflammatory syndrome associated with SARS-CoV-2 infection in 45 children: a first report from Iran. Epidemiol Infect. 2020;148:e196. https://doi.org/10.1017/ S095026882000196X

11. Huh HJ, Hong KH, Kim TS, Song SH, Roh KH, Lee H, et al. Surveillance of coronavirus disease 2019 (COVID-19) testing in clinical laboratories in Korea. Ann Lab Med. 2021;41:225-9. https:/ / doi.org/10.3343/alm.2021.41.2.225

12. Miller J, Cantor A, Zachariah P, Ahn D, Martinez M, Margolis KG. Gastrointestinal symptoms as a major presentation component of a novel multisystem inflammatory syndrome in children that is related to coronavirus disease 2019: a single center experience of 44 cases. Gastroenterology. 
2020;159:1571-1574.e2. https:/ / doi.org/10.1053/ j.gastro.2020.05.079

13. Tullie L, Ford K, Bisharat M, Watson T, Thakkar H, Mullassery D, et al. Gastrointestinal features in children with COVID-19: an observation of varied presentation in eight children. Lancet Child Adolesc Health. 2020;4:e19-20. https:/ / doi.org/10.1016/S2352-4642(20)30165-6

14. Aziz M, Haghbin H, Lee-Smith W, Goyal H, Nawras A, Adler DG. Gastrointestinal predictors of severe COVID-19: systematic review and meta-analysis. Ann Gastroenterol. 2020;33:615-30
15. Fabi M, Corinaldesi E, Pierantoni L, Mazzoni E, Landini C, Bigucci B, et al. Gastrointestinal presentation of Kawasaki disease: A red flag for severe disease? PLoS One. 2018;13:e0202658. https://doi.org/10.1371/journal. pone. 0202658

Address for correspondence: Eun Hwa Choi, Professor of Pediatrics, Seoul National University College of Medicine, 101 Daehak-ro, Jongno-gu, Seoul 110-769, South Korea; email: eunchoi@snu.ac.kr
Clinical Characteristics of Disseminated Strongyloidiasis, Japan, 1975-2017

- Epidemiology of Cryptosporidiosis, New York City, New York, USA, 1995-2018

- Public Health Response to Tuberculosis Outbreak among Persons Experiencing Homelessness, Minneapolis, Minnesota, USA, 2017-2018

- Mycobacterium tuberculosis Complex Lineage 3 as Causative Agent of Pulmonary Tuberculosis, Eastern Sudan

- Norovirus Outbreak Surveillance, China, 2016-2018

- Methicillin-Resistant Staphylococcus aureus Bloodstream Infections and Injection Drug Use, Tennessee, USA, 2015-2017

- Randomized Trial of 2 Schedules of Meningococcal B Vaccine in Adolescents and Young Adults, Canada

- Human Immune Responses to Melioidosis and Cross-Reactivity to Low-Virulence Burkholderia Species, Thailand

- Role of Live-Duck Movement Networks in Transmission of Avian Influenza, France, 2016-2017

- Multidrug- and Extensively DrugResistant Mycobacterium tuberculosis Beijing Clades, Ukraine, 2015

- Stable and Local Reservoirs of Mycobacterium ulcerans Inferred from the Nonrandom Distribution of Bacterial Genotypes, Benin

- Suspected Locally Acquired Coccidioidomycosis in Human, Spokane, Washington, USA

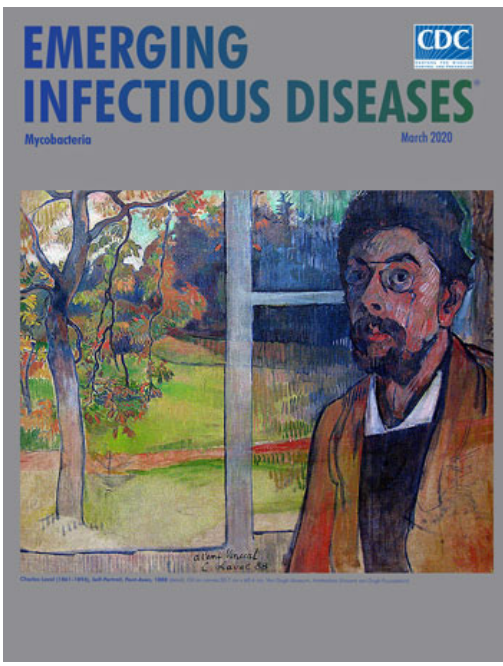

- US Tuberculosis Rates among Persons Born Outside the United States Compared with Rates in Their Countries of Birth, 2012-2016

- Genomic and Phenotypic Variability in Neisseria gonorrhoeae Antimicrobial Susceptibility, England

- High Prevalence of and Risk Factors for Latent Tuberculosis Infection among Prisoners, Tianjin, China

- Risk Factors for Complicated Lymphadenitis Caused by Nontuberculous Mycobacteria in Children

- Pregnancy Outcomes among Women Receiving rVSVA-ZEBOV-GP Ebola Vaccine during the Sierra Leone Trial to Introduce a Vaccine against Ebola

- Pulmonary Nocardia ignorata Infection in Gardener, Iran, 2017
- Acquisition of Plasmid with Carbapenem-Resistance Gene bla ${ }_{\mathrm{KPC}}$ in Hypervirulent Klebsiella pneumoniae, Singapore

- Long-Term Rodent Surveillance after Outbreak of Hantavirus Infection, Yosemite National Park, California, USA, 2012

- Mycobacterium tuberculosis Beijing Lineage and Risk for Tuberculosis in Child Household Contacts, Peru

- Human Exposure to Hantaviruses Associated with Rodents of the Murinae Subfamily, Madagascar

- Avian Influenza Virus Detection Rates in Poultry and Environment at Live Poultry Markets, Guangdong, China

- Diphtheria Outbreaks in Schools in Central Highland Districts, Vietnam, 2015-2018

- Progressive Vaccinia Acquired through Zoonotic Transmission in a Patient with HIV/AIDS, Colombia

- Mycobacterium senegalense Infection after Implant-Based Breast Reconstruction, Spain

- Low Prevalence of Mycobacterium bovis in Tuberculosis Patients, Ethiopia

- Metagenomics of Imported Multidrug-Resistant Mycobacterium leprae, Saudi Arabia, 2017

- Coccidioidomycosis Skin Testing in a Commercially Insured Population, United States, 2014-2017

- Need for BCG Vaccination to Prevent TB in High-Incidence Countries and Populations

\section{EMERGING} INFECTIOUS DISEASES 